\title{
Success Factors in Decision Making and Negotiation Processes for Raw Material Supply Transactions
}

Mag. Alexander Stelzer

LLM, Doctoral Student, University of Riga, Latvia

Abstract

Problem Statement: The topics raw materials, waste and energy will be the top economic issues of the future, or influence and affect all other subjects (human resources, culture, society, etc. ); all combined with technology, IT and Tech Communication. The shortage of raw materials is one of the central issues in the future perspective and this fact is already influencing the relationship between buyer, producer and supplier.

Keywords: Success, factors, decision making, negotiation processes, raw material, supply, transactions

\section{Introduction}

\section{Theoretical Framework}

The interactions between purchasers, suppliers and producers respect to the procurement of raw materials, goods and products are examined specifically in terms of their economic and psychological satisfaction. Is it possible to satisfy all parties not only in an economical way, butalso in their mental satisfaction. Harvard Negotiation Concept plus Transparency

Future Main Themes of Business Life

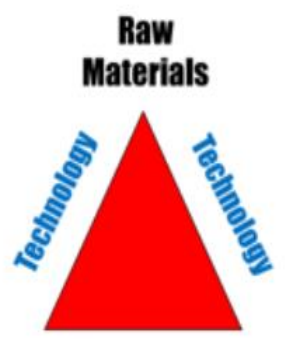

Waste Technology Energy

\section{$\Rightarrow$ Future Business Triangle of today and tomorrow}




\section{Trading Situation}

\section{Situation Poster}

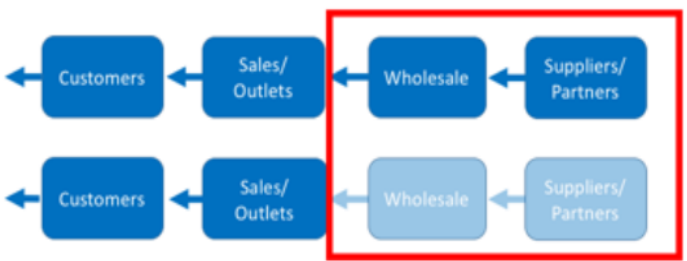

Task:

Evaluation of the tasks and problems between wholesalers and suppliers / partners and the creation of future models and associated tools, because of the greatest need;

$\rightarrow$ Creation of a "toolbox" in the scientific context for real application.

\section{Research Questions}

Question 1: $\bullet$ How to run negotiations between purchasers, suppliers and producers, so that both parties are really satisfied (economically and mentally)?

Question 2: - Must the negotiations be transparent in the relation to prices, quantities, etc., so the way of approaching the interests will increase consequently the satisfaction?

\section{Model}

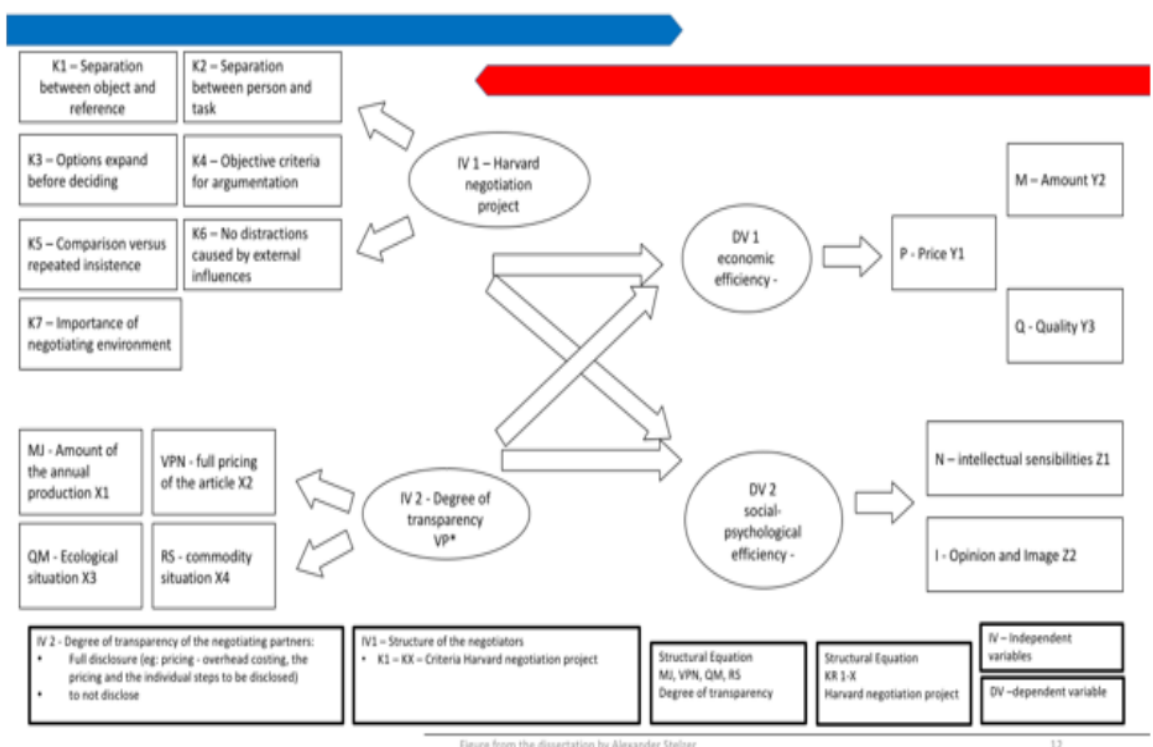




\section{Hypotheses}

Hypothesis $\mathrm{H} 1$ : The structure of the negotiation process has an impact on the outcome of negotiations

Hypothesis $\mathrm{H} 2$ : The more transparent the negotiation process, the more efficient the negotiation efficiency from the perspective

\section{Method}

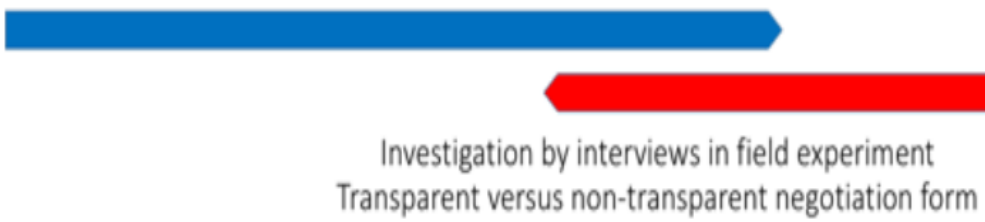

\section{Supplier}

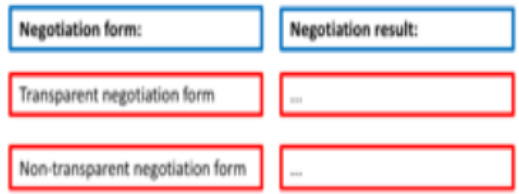

Reality of negotiation:

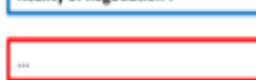

\section{Result of negotiation}

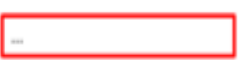

\section{Acquirer}

Negotiation form:

Transparent negotiation form

Non transparent negotiation form

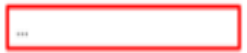

Reality of negotiation:

Result of negotiation

\section{Conclusion}

Economic and mental satisfaction for all negotiating parties means an consequent extension of the Harvard concept with the level of transparency.

If we could resolve the "fronts" between purchasers, suppliers and producers to a cooperation in the form of a transparency , this would help the entire cycle.

(Note. This article has been adapted from the poster the author provided - Editor) 